\title{
FTO genotype and weight loss: systematic review and meta-analysis of 9563 individual participant data from eight randomised controlled trials
}

\begin{abstract}
Katherine M Livingstone, ${ }^{1,2}$ Carlos Celis-Morales, ${ }^{1,3}$ George D Papandonatos, ${ }^{4}$ Bahar Erar, ${ }^{4}$ Jose C Florez,, ${ }^{5,6}$ Kathleen A Jablonski,7 Cristina Razquin, ${ }^{8,9}$ Amelia Marti, ${ }^{9,10}$ Yoriko Heianza, ${ }^{11}$ Tao Huang, ${ }^{11,12}$ Frank M Sacks, ${ }^{13}$ Mathilde Svendstrup, ${ }^{14,15}$ Xuemei Sui, ${ }^{16}$ Timothy S Church, ${ }^{17}$ Tiina Jääskeläinen, ${ }^{18,19}$ Jaana Lindström, ${ }^{20}$ Jaakko Tuomilehto, ${ }^{21,22}$ Matti Uusitupa, ${ }^{18}$ Tuomo Rankinen, ${ }^{23}$ Wim H M Saris, ${ }^{24}$ Torben Hansen,, ${ }^{14}$ Oluf Pedersen, ${ }^{14}$ Arne Astrup, ${ }^{25}$ Thorkild I A Sørensen, ${ }^{14,26}$ Lu Qi,,11,13 George A Bray, ${ }^{17}$ Miguel A Martinez-Gonzalez,9,10 J Alfredo Martinez,, 9,10,27 Paul W Franks,, 13,28 Jeanne M McCaffery, ${ }^{29}$ Jose Lara, ${ }^{1,30}$ John C Mathers'
\end{abstract}

\section{ABSTRACT}

OBJECTIVE

end of article.

Correspondence to:

J C Mathers

john.mathers@newcastle.ac.uk Additional material is published online only. To view please visit the journal online.

Cite this as: $B M$ J 2016;354:14707 http://dx.doi.org/10.1136/bmj.i4707

Accepted: 17 August 2016

\section{WHAT IS ALREADY KNOWN ON THIS TOPIC}

Obesity is a major public health burden and its prevalence is increasing worldwide The minor allele for the fat mass and obesity associated gene (FTO) rs9939609 is linked to increased risk of obesity

\section{WHAT THIS STUDY ADDS}

Carriage of the FTO minor allele has no effect on the efficacy of lifestyle and drug related weight loss interventions ethnicity, sample size, sex, and baseline body mass index and age category.

\section{CONCLUSIONS}

We have observed that carriage of the FTO minor allele was not associated with differential change in adiposity after weight loss interventions. These findings show that individuals carrying the minor allele respond equally well to dietary, physical activity, or drug based weight loss interventions and thus genetic predisposition to obesity associated with the FTO minor allele can be at least partly counteracted through such interventions.

SYSTEMATIC REVIEW REGISTRATION PROSPERO CRD42015015969.

\section{Introduction}

The epidemic of obesity, together with its associated health burden, continues to spread globally. ${ }^{1}$ With an estimated 2.1 billion adults now overweight or obese, ${ }^{2}$ there is an urgent need to develop more effective strategies for preventing and managing obesity. ${ }^{34}$ Genotype plays an important role in the development of obesity, ${ }^{5}$ and recent genome wide association studies have identified multiple loci associated with body mass index ${ }^{67}$ and distribution of body fat. ${ }^{8}$ Specifically, 97 loci have been identified as accounting for about $2.7 \%$ of variation in body mass index, ${ }^{6}$ of which the fat mass and obesity associated (FTO) gene, ${ }^{9}$ melanocortin 4 receptor (MC4R) gene, ${ }^{10}$ and transmembrane protein 18 (TMEM18) gene ${ }^{11}$ have shown the strongest associations. Supplementary appendix 1 summarises the details of key genes associated with body mass index. However, so far the FTO gene explains the largest amount of the genetic variance in obesity traits over the lifespan. ${ }^{9}$ Those homozygous for the FTO (rs9939609) minor allele weigh on average $3 \mathrm{~kg}$ more and have a 1.7fold increased odds of being obese compared with those homozygous for the lower risk allele. ${ }^{12}$

To date, several intervention studies have explored the interaction between FTO genotype and lifestyle changes on adiposity. ${ }^{13-19}$ A study in 742 obese adults found that after a two year dietary intervention, those with the FTO minor allele had a $1.5 \mathrm{~kg}$ greater weight loss on a high protein diet but not on a low protein diet. ${ }^{16}$ Similarly, a six month moderate intensity 
exercise intervention in 105 obese women resulted in up to twofold greater weight loss in carriers of the FTO minor allele compared with non-carriers. ${ }^{14}$ In contrast, a one year lifestyle intervention in 3548 adults showed smaller changes in adiposity in those with the FTO minor allele compared with those without, ${ }^{20}$ and a lifestyle intervention in 502 participants found no effect of the FTO genotype on weight loss. ${ }^{21}$ Moreover, an analysis of data from the Diabetes Prevention Programme (DPP) $(n=1824)$ and the Action for Health in Diabetes study (Look AHEAD; $n=3906$ ), showed no effect of FTO genotype on weight loss. ${ }^{22}$

A recent meta-analysis by Xiang et $\mathrm{al}^{23}$ assessed the effect of the FTO genotype on weight change across several randomised and non-randomised intervention studies. In the randomised controlled trials the authors averaged FTO effects on weight change across both intervention and control arms of the studies under investigation and concluded that homozygous carriers of the FTO minor allele lost $0.44 \mathrm{~kg}$ (95\% confidence interval 0.09 to $0.79 \mathrm{~kg}$ ); $\mathrm{P}=0.015$ ) more weight than non-carriers. ${ }^{23}$ However, such averaging across study arms can be justified only in the absence of single nucleotide polymorphisms by treatment interactions, which were not tested by Xiang et al. ${ }^{23}$ This limitation, combined with the authors' use of summary level data to assess the influence of participant level characteristics-eg, age and body mass index at baseline, means that the findings are potentially misleading and subject to aggregation bias. ${ }^{23}$

We carried out a systematic review and meta-analysis to provide a critical analysis of the evidence that the FTO genotype influences changes in obesity related outcomes in randomised weight loss interventions. In particular, we investigated whether the FTO genotype (rs9939609 or a proxy) predicted the magnitude of weight loss in response to a randomised weight loss programme. This analysis also employed individual participant data analyses to assess the role of participant level covariates.

\section{Methods}

Our systematic review was conducted according to Cochrane ${ }^{24}$ and the Centre for Reviews and Dissemination guidelines ${ }^{25}$ and is reported according to the preferred reporting items for systematic reviews and meta-analyses guidelines. ${ }^{26}$ The protocol was registered with PROSPERO, the International Prospective Register of Systematic Reviews (CRD42015015969). An initial systematic literature search was undertaken to identify eligible studies for inclusion in the meta-analysis. Once eligible studies were identified, we invited the corresponding authors of those studies to contribute individual participant data or to undertake a prescribed statistical plan (if individual participant data were not available for sharing). If authors did not reply, we excluded the corresponding studies from the meta-analysis but reported the study characteristics.

\section{Search strategy}

We undertook an electronic search to identify intervention studies reporting weight loss by FTO genotype (rs9939609 or a proxy) after a dietary, physical activity, or drug based intervention. Ovid Medline (www.nlm. nih.gov/bsd/pmresources.html), Embase (www. embase.com), and Scopus (www.scopus.com) were searched systematically for studies published from inception to November 2015. To determine other studies potentially eligible for inclusion we hand searched the reference lists of identified publications and previously published related systematic reviews. The search strategy involved combining words from the concepts of genes, weight loss, and highly sensitive search filters for identifying randomised controlled trials for Ovid Medline and Embase ${ }^{24} 27$ (see supplementary appendix 2 for the detailed search strategy).

\section{Study selection criteria}

Included studies were randomised intervention studies in overweight or obese (body mass index $\geq 25$ ) participants aged 18 years or more designed to induce weight loss (either as a primary or a secondary outcome) and that reported change in adiposity indices (body mass index, body weight, or waist circumference) by FTO genotype (rs9939609 or a proxy). Studies in non-overweight or non-obese participants (body mass index $<25$ ) and children ( $<18$ years) were excluded. Only publications with an English language abstract were included. We included studies on men and women with or without health risk factors (such as raised blood pressure, abnormal lipid levels, and metabolic syndrome).

\section{Data extraction}

Two reviewers (KML and JL) independently assessed publications for eligibility. The decision to include studies was hierarchical and made initially on the basis of the study title and abstract; when a study could not be excluded with certainty, the full text was obtained for evaluation. Discrepancies between reviewers were resolved through discussion with a third reviewer (CCM), and a consensus approach was used. Information extracted from studies included study design (intervention type (dietary, physical activity, or drug intervention), length of follow-up, and country); participant characteristics (age, sex, and ethnicity); description of measurement methods; and information to assess the risk of bias. Study quality was assessed using the Cochrane risk of bias tool (see supplementary appendix 3). ${ }^{24}$ Two reviewers extracted data, one independently and the second confirming or completing information required.

\section{Statistical analysis}

Statistical analyses were conducted using Stata 14.0 software (Stata, College Station, TX) and Review Manager (RevMan Version 5.1 for Windows Copenhagen; Nordic Cochrane Centre, Cochrane Collaboration, 2015). A two step individual participant data analysis was undertaken, whereby individual data were first analysed separately by study and subsequently aggregated using weighted meta-analyses. ${ }^{28} \mathrm{~A}$ common analytical plan was used for all studies under investigation, with covariate coding matched across studies as closely as possible. We checked summary level data in the log 
files returned by researchers for consistency with the prescribed statistical plan. To ensure consistency in the pattern of results we compared all individual participant data analyses with the published studies.

Outcomes of interest were change in body mass index, body weight, or waist circumference between baseline and follow-up (change calculated as follow-up measurement minus baseline measurement). Linear regression analyses assessed change in the dependent variable (change in body weight, body mass index, or waist circumference) by FTO genotype (independent variable) separately by study arm, with FTO genotype coded using an allele-dose model (ie, number of copies of the minor allele). We adjusted arm specific models for age (continuous), sex, baseline outcome (body mass index, body weight, or waist circumference; continuous), ethnicity (categorical), country or centre (categorical), socioeconomic status (categorical), physical activity (continuous where possible), and smoking (categorical; see supplementary appendix 4). Participants were excluded from the analyses if they did not have complete data for all outcomes and covariates.

Table 2 presents two sets of regression coefficients. The first set of coefficients is arm specific and captures within arm change in the outcome during the course of the study for each copy of the FTO minor allele. Both treatment and control arms are included in these calculations. The second set of coefficients is intervention specific and captures mean differences in FTO allelic effects between treatment and control arms, where a negative coefficient indicates that individuals carrying a minor allele had a greater reduction in the outcomes of interest (weight, body mass index, or waist circumference) after the intervention than those without the minor allele. So, for studies with $\mathrm{K}$ treatment arms and a single control arm, $\mathrm{K}+1$ coefficients are used to capture FTO effects on within arm change scores (set 1), and K coefficients are used to capture FTO effects on treatment versus control differences in change scores (set 2). Importantly, the first set of coefficients is estimated from separate groups of study participants, rendering them statistically independent and facilitating standard error calculations for the second set of coefficients that capture the interaction effects of gene $\mathrm{x}$ treatment. However, the second set of coefficients uses a common control group for each study, which introduces dependence at the study level.

This second set of regression coefficients from table 2 was used to evaluate differences in FTO allelic effects on the outcomes of interest between intervention and control groups. We used random effects models to estimate the pooled effect sizes and to account for both sampling error and between study variation in population. ${ }^{29}$ Meta-estimates were weighted by the inverse of the variance of the effect size (that is, 1 divided by variance), where variance took into account the two potential sources of variation (ie, variance within and between studies).

For studies with more than one active treatment arm and a single control arm, including all differences in treatment versus control in the meta-analysis shown in figure 2 would have invalidated the standard error calculations programmed into our meta-analytical packages, as these packages assume independent intervention effects, not ones based on comparing different treatment arms with a shared control group. For such multi-arm studies, we used a fixed effect, inverse variance meta-analysis to produce an overall estimate of FTO effects across all treatment arms, in the absence of within study heterogeneity. We then compared that overall FTO effect across treatment arms with the FTO effect on the placebo arm, thus creating a single intervention versus control comparison for each study under consideration that satisfied statistical independence assumptions underlying standard error calculations. This method is recommended by Higgins et $\mathrm{al}^{30}$ to avoid excessive weightings from "double counts" originating from the control group shared by the multiple treatment arms.

A further complication arose because our meta-analytical packages required that intervention effects be entered in terms of mean differences, their standard deviation, and sample size. As the meta-analyses returned point estimates and their standard errors instead, we arbitrarily fixed our effective sample size for the combined intervention effect to the sum of the sample sizes of all $\mathrm{K}$ treatment groups in each study, and then back calculated the standard deviation required for the combined intervention effect to have the right level of precision. As the forest plot depends solely on the product of the standard deviation with the square root of the reported sample size, the arbitrariness in these calculations did not affect the validity of the forest plot itself.

Heterogeneity between studies was evaluated using the $\mathrm{I}^{2}$ test and Galbraith plots ${ }^{31}$ and the $95 \%$ confidence interval for $\mathrm{I}^{2}$ calculated using the method of Higgins et al. ${ }^{3233}$ Small study effects were appraised by visual inspection of funnel plots of effect size against the standard error, with asymmetry assessed formally with Egger's test, chosen over Begg's test for its greater specificity and power, ${ }^{34}$ where a $P$ value less than 0.1 was considered as significant. ${ }^{35}$

To explore potential sources of heterogeneity, we conducted moderation testing using intervention type (diet and diet and/or exercise), intervention length ( $\leq 6$ months and $>6$ months), age ( $<50$ years and $\geq 50$ years), sex (binary), body mass index ( $<30$ and $\geq 30$ ), and race/ethnicity (white, black or African-American, and Hispanic) as putative categorical moderators. Between stratum comparisons in table 3 were used to test the hypothesis that the relation between FTO genotype and intervention effects on change in obesity related outcomes differed across levels of the moderator, whereas within stratum tests helped elucidate the nature of the interaction, if present. Variables employed as stratification factors were removed as covariates from the corresponding regression model. In addition, we investigated intervention length as a continuous variable in moderation analyses.

As a secondary analysis, we recoded FTO genotypes using a dominant model, where participants with one (AT) and two (AA) copies of the minor allele were grouped together and compared with those with no copies of the minor allele (TT). 
Given that only one study ${ }^{20}$ included drug based interventions, we run a further sensitivity analysis where only the lifestyle intervention treatment arm was included. In a final set of sensitivity analysis, we removed from the analyses the study that contributed the most weight to the analyses (Look AHEAD).

To investigate whether changes in obesity outcomes were driven by associations between obesity outcomes at baseline and FTO genotype, additional linear regression analyses (presented in supplementary appendix 8) assessed relations between baseline values of the dependent variable (body weight, body mass index, or waist circumference) and FTO genotype (independent variable), where the FTO genotype was coded using an allele-dose model. These analyses were adjusted for age, sex, ethnicity, country or centre, socioeconomic status, physical activity, and smoking and aggregated using a fixed effects, inverse variance meta-analysis.

\section{Patient involvement}

No patients were involved in setting the research question or the outcome measures, nor were they involved in developing plans for design or implementation of the study. No patients were asked to advise on interpretation or writing up of results. There are no plans to disseminate the results of the research to study participants or the relevant patient community.

\section{Results}

Searches yielded 2247 publications after removal of duplicates. One additional record was identified through other sources. Following screening ( $n=2248$ ), 2220 publications were excluded based on the titles and abstracts and 28 full text articles were assessed for eligibility (fig 1). Full text articles were excluded from the qualitative synthesis because either they presented

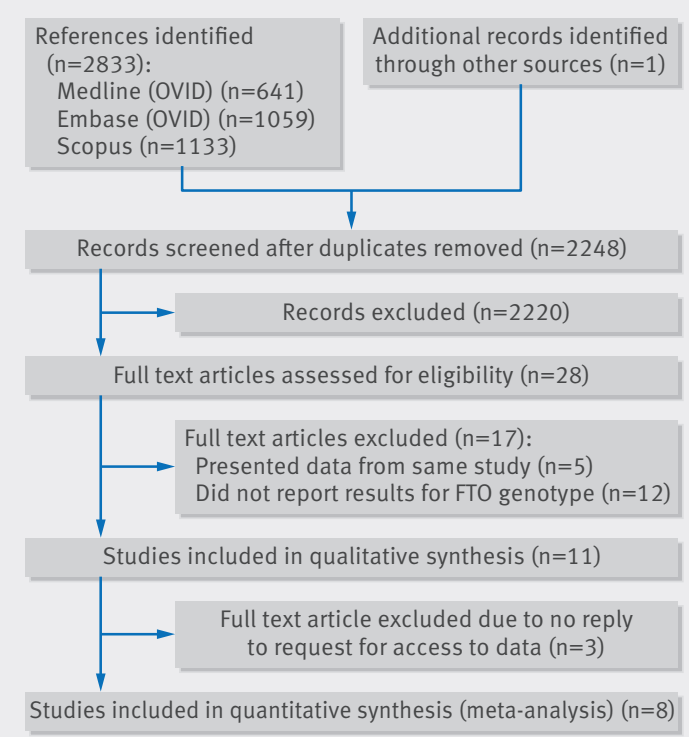

Fig 1 | Study selection flow diagram based on preferred reporting items for systematic reviews and meta-analyses statement data from the same study $(n=5)$ or they did not report results for FTO genotype $(n=12)$. A total of 11 studies were eligible for inclusion in the qualitative synthesis. Three studies were excluded from the quantitative synthesis (meta-analysis) because the authors did not reply to our request for access to data. A total of eight studies were included in the present meta-analysis. ${ }^{13-2136}$ Individual participant data were analysed from all eight studies: five studies provided individual participant data $^{1314172136}$ and three studies performed our prescribed statistical analysis and provided summary level data. ${ }^{15} 1620$

\section{Study characteristics}

The pooled population of the 11 studies eligible for inclusion in the qualitative synthesis comprised 10000 adults (table 1). After exclusion of participants without full data for outcomes and covariates (see supplementary appendix 5), the pooled population of adults included in the quantitative synthesis (meta-analysis) was 9563. The mean age at baseline was 51.6 (range 28-74) years and the mean body mass index was 32.2 (23.8-43.2). All studies provided data for single nucleotide polymorphism rs9939609, with the exception of the Dose Response to Exercise in Women aged 45-75 years (DREW) study, ${ }^{14}$ where rs8050136 was studied and is known to be in high linkage disequilibrium with rs9939609 (HapMap US residents of European ancestry: $\left.r^{2}>0.84\right)$. Nine studies were in men and women and two studies in women only. Five studies were of dietary interventions, three of dietary and exercise based interventions, one of an exercise only intervention, and two of a drug and/or lifestyle intervention. The duration of follow-up ranged from eight weeks to three years. Studies were conducted in North America, South America, and Europe; six studies were in white participants and four in mixed populations (table 1).

\section{Study quality and small study effects}

No studies were excluded from the meta-analysis based on quality assessment (see supplementary appendix 3). Visual inspection of funnel plots (see supplementary appendix 6) and Galbraith plots (see supplementary appendix 7) did not identify any systematic small study effects. Similarly, no significant bias was observed for body mass index $(\mathrm{P}=0.55)$, body weight $(\mathrm{P}=0.69)$, or waist circumference $(\mathrm{P}=0.39)$.

\section{Assessment of gene $x$ treatment interaction effects on change in obesity related outcomes}

Table 2 summarises the minor allele effects (coefficient and standard error) on obesity outcomes after the weight loss intervention for each study and study arm. Table 2 also presents differences in minor allele effects between treatment and control arms, indicative of gene by treatment interactions. Mean treatment versus control differences in body mass index change for each copy of the FTO minor allele ranged from -0.33 (95\% confidence interval -1.13 to 0.47$)$ after six month follow-up in DREW to 0.14 (-0.06 to 0.35) after one year in Look AHEAD. Body mass index was reduced by an 


\begin{tabular}{|c|c|c|c|c|c|c|c|c|c|c|c|}
\hline \multirow[b]{2}{*}{ Study } & \multicolumn{3}{|c|}{ No of participants } & \multirow[b]{2}{*}{ SNP } & \multirow[b]{2}{*}{ MAF } & \multicolumn{2}{|l|}{ Intervention } & \multirow[b]{2}{*}{ Region } & \multirow[b]{2}{*}{ Ethnicity } & \multicolumn{2}{|l|}{ Mean (SD) } \\
\hline & All & Men & Women & & & Type & Length & & & Age (years) & BMI \\
\hline DPP20 & 2835 & 962 & 1873 & rs9939609 & 40.9 & Drug and lifestyle & 1 year & North America & Mixed & $51.0(10.6)$ & $34.0(6.7)$ \\
\hline DREW $^{14}$ & 278 & - & 278 & rs8050136* & 41.0 & Exercise & 6 months & North America & Mixed & $57.3(6.7)$ & $31.8(3.8)$ \\
\hline Finnish DPS 21 & 264 & 97 & 167 & rs9939609 & 41.5 & Diet and exercise & 3 years & Europe & White & $54.8(7.2)$ & $31.1(4.6)$ \\
\hline Food4Me ${ }^{36}$ & 671 & 313 & 358 & rs9939609 & 44.3 & Diet and exercise & 6 months & Europe & White & $43.3(12.5)$ & $27.8(4.7)$ \\
\hline Look AHEAD 15 & 3637 & 1601 & 2036 & rs9939609 & 44.5 & Diet and exercise & 1 year & North America & Mixed & $59.1(6.9)$ & $36.2(6.0)$ \\
\hline POUNDS LOST ${ }^{16}$ & 600 & 240 & 360 & rs9939609 & 45.1 & Diet & 2 years & North America & White & $51.6(9.1)$ & $32.6(3.9)$ \\
\hline PREDIMED ${ }^{13}$ & 735 & 335 & 400 & rs9939609 & 41.6 & Diet & 3 years & Europe & White & $67.6(6.0)$ & $29.2(3.3)$ \\
\hline NUGENOB ${ }^{17}$ & 543 & 136 & 407 & rs9939609 & 41.5 & Diet & 10 weeks & Europe & White & $36.8(7.9)$ & $35.7(5.0)$ \\
\hline Ramos et $\mathrm{al}^{37}$ & 86 & - & 86 & rs9939609 & $\mathrm{NE}$ & Drug & 6 months & South America & White & $51(3.0)$ & $26.6(2.8)$ \\
\hline De Luis et $\mathrm{al}^{38}$ & 305 & 80 & 225 & rs9939609 & 44.1 & Diet & 3 months & Europe & $\mathrm{NE}$ & $43.5(15.3)$ & $36.6(6.6)$ \\
\hline MOVE!39 & 46 & 33 & 13 & rs9939609 & $\mathrm{NE}$ & Diet & 8 weeks & North America & Mixed & NE & $\mathrm{NE}$ \\
\hline
\end{tabular}

additional -0.02 ( -0.13 to 0.09$) ; \mathrm{I}^{2} 0$ (95\% confidence interval 0 to $68 ; \mathrm{P}=0.69)$, body weight by $-0.04(-0.34$ to $0.26) \mathrm{kg} ; \mathrm{I}^{2} 0$ ( 0 to $68 ; \mathrm{P}=0.78$ ), and waist circumference by -0.06 ( -0.43 to 0.31$) \mathrm{cm} ; \mathrm{I}^{2} 0$ ( 0 to $68 ; \mathrm{P}=0.75$ ) for each copy of the FTO minor allele after weight loss intervention compared with that expected under naturalistic change in the control group (fig 2). When using a dominant FTO genotype model (AA/AT $v$ TT), body mass index, body weight, and waist circumference were reduced by 0.05 ((95\% confidence interval -0.21 to 0.11$)$; $\mathrm{P}=0.558), 0.15$ ((-0.60 to 0.30$) \mathrm{kg} ; \mathrm{P}=0.524)$, and 0.22 $((-0.77$ to 0.33$) \mathrm{cm}$; $\mathrm{P}=0.437)$ after intervention in carriers of the minor allele compared with non-carriers.

\section{Stratified and sensitivity analyses}

The relation between FTO genotype and differences between treatment and control in change in obesity related outcomes (body mass index, body weight, and waist circumference) after weight loss interventions was not influenced by study type, study length, sex, race/ethnicity, or body mass index category (table 3). Change in body mass index for each copy of the FTO minor allele was significant in participants aged 50 or more years ( $\beta-0.23$ (95\% confidence interval -0.44 to $-0.22) ; \mathrm{I}^{2} 0$ (95\% confidence interval 0 to 75$)$; $\mathrm{P}=0.03$ ), but not in participants aged less than 50 years $(\beta-0.04$ ( -0.26 to 0.18$) ; \mathrm{I}^{2} 0$ (0 to 75$)$; $\left.\mathrm{P}=0.75\right)$; however, the corresponding moderation test failed to attain significance $(\mathrm{P}=0.21)$. In addition, intervention length modelled as a continuous variable did not significantly affect the relation between FTO genotype and differences between treatment and control in change in obesity related outcomes (body mass index, body weight, and waist circumference) after weight loss interventions.

Given the presence of a gene $\mathrm{x}$ treatment interaction on waist circumference in table 3 between metformin and control arms in study DPP, we investigated exclusion of the metformin and troglitazone arms from DPP. This did not change the pattern of results (data not shown).

After removal of Look AHEAD, the largest study, overall effect sizes for body mass index, body weight, and waist circumference in figure 2 changed to $-0.09(-0.22$ to $0.04 ; \mathrm{P}=0.17),-0.21(-0.56$ to 0.14$) \mathrm{kg} ; \mathrm{P}=0.25)$, and -0.01 ( -0.45 to 0.42$) \mathrm{cm} ; \mathrm{P}=0.96)$, respectively, but failed to attain statistical significance.

\section{Baseline association testing}

To facilitate better interpretation of any effect of FTO genotype on weight loss after an intervention, we investigated the association between FTO genotype and obesity measures at baseline. FTO genotype differences in body mass index, body weight, and waist circumference at baseline for each study are presented in supplementary appendix 8. Collectively, for each copy of the FTO minor allele, body mass index, body weight, and waist circumference increased by 0.31 (0.14 to 0.47 ); $\mathrm{P}<0.001), 0.89$ (0.45 to 1.32) kg; $\mathrm{P}<0.001)$, and 0.63 (0.28 to 0.98$) \mathrm{cm} ; \mathrm{P}<0.001)$, respectively.

\section{Discussion}

The present systematic review and meta-analysis used individual participant data to investigate the differential effect of FTO genotype on response to weight loss intervention in randomised controlled trials. Our meta-analysis of eight studies involving 9563 adults showed that carriage of the FTO minor allele does not influence change in adiposity measures in response to weight loss intervention, compared with treatment controls. Since we observed a strong association of the FTO minor allele with greater adiposity at baseline, this neutral effect of FTO minor allele on weight loss is an important finding for the development of effective weight loss interventions in the context of the global epidemic of obesity. Specifically, people who carry obesity risk FTO genotypes respond equally well to weight loss treatment.

Our finding of the lack of a FTO genotype by treatment interaction on change in obesity related outcomes expands on a recent meta-analysis on the effect of FTO genotype on weight loss, ${ }^{23}$ which analysed FTO genotypic effects on weight change averaged across highly effective treatment arms and control arms that were designed to produce minimal to no weight change. Unlike Xiang et al, we included only randomised controlled trials, which provide stronger evidence than 


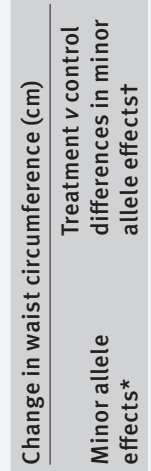

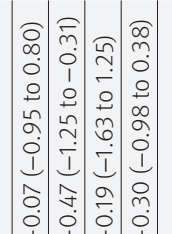
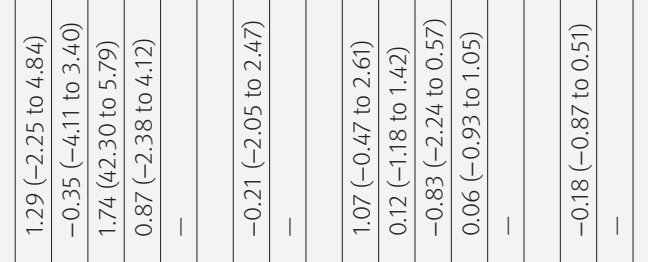

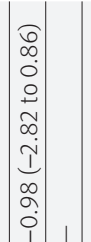
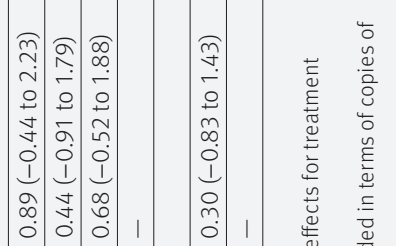

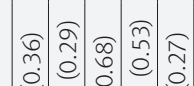

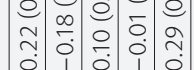

ת.

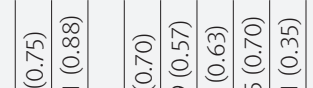

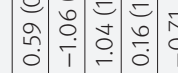

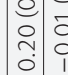

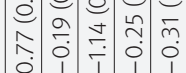

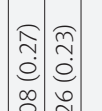

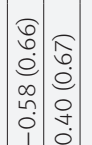

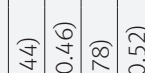

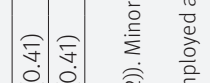

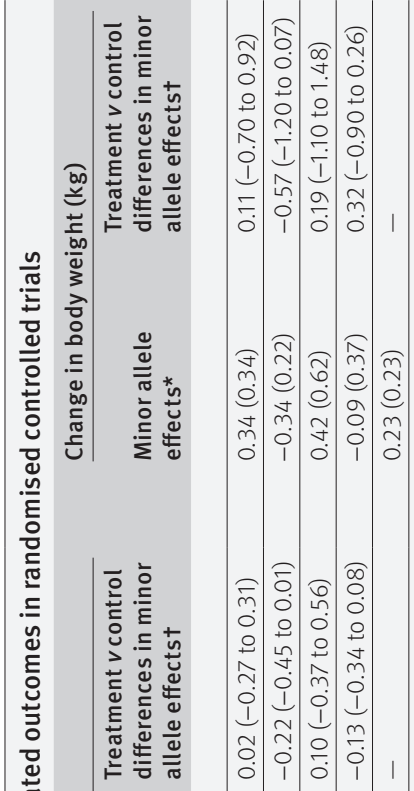

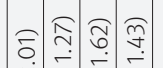

ن.

空空

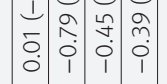

每

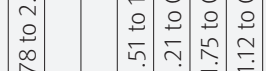

(1)

|

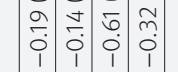

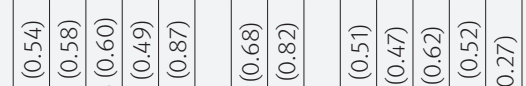

:

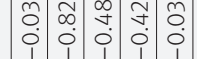

ít.

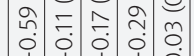

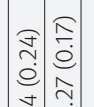

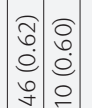

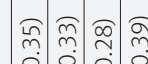

จุ

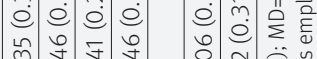

$\begin{array}{llll}1 & 1 & i & 0 \\ 0 & 0 & 0 & 0\end{array}$

i:

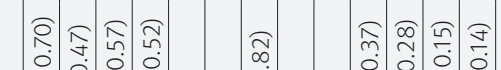

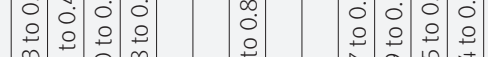

$=0$

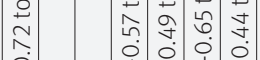

드을

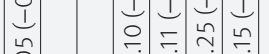

$\begin{array}{llll}i & i & i & i\end{array}$

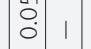

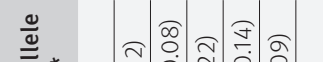

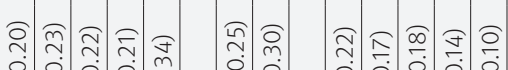

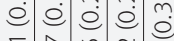

o

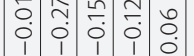

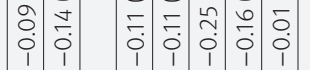

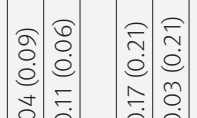

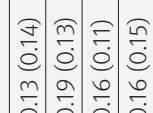

을

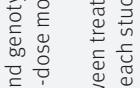

ธ

苋

$\infty$
$\infty$

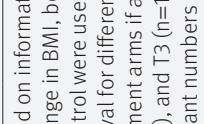

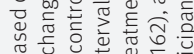

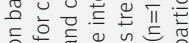

先

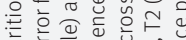

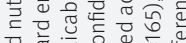

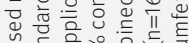

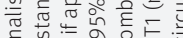

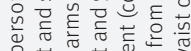

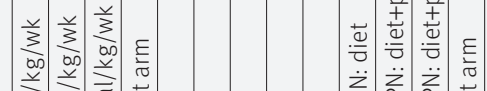

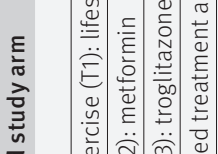

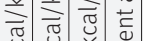

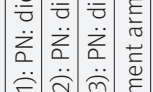

구에

Eิ

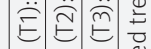

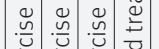

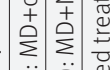

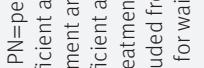

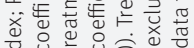

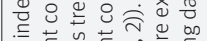

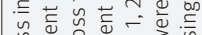




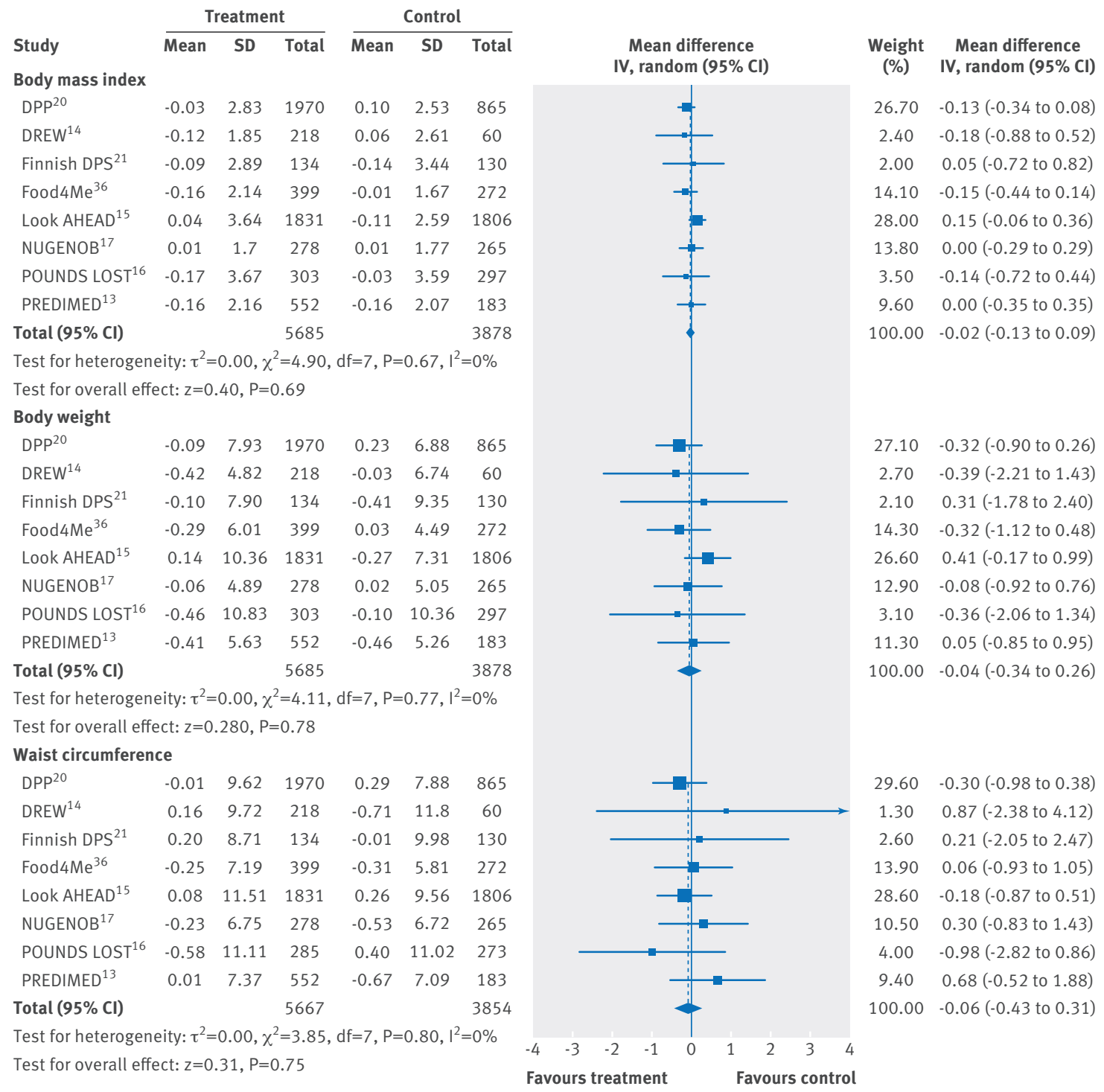

Fig 2 | Forest plot of change in body mass index, body weight, and waist circumference after weight loss intervention for each copy of the FTO minor allele (rs9939609 genotype or a proxy) in treatment versus control arm in random effects meta-analysis of 9563 adults. Values for treatment and control represent coefficient and standard deviation from linear regression analyses adjusted for age, sex, baseline outcome (body mass index, body weight, or waist circumference), ethnicity, country or centre, socioeconomic status, physical activity, and smoking where appropriate. When more than one treatment arm was present, values represent combined effects across treatment arms

non-randomised trials and limited within study bias and between study heterogeneity. Of the 10 studies included by Xiang et al, six overlapped with studies included in the present meta-analysis, ${ }^{13-1721}$ with the remaining studies being either randomised controlled trials ${ }^{40}$ where the authors did not agree to provide individual level data for the present analysis $(n=1)$ or non-randomised controlled trials $(n=3) . .^{41-43}$ Moreover, we examined whether the effect of FTO genotype differed among those randomised to weight loss interventions compared with those randomised to control groups. This was achieved by building on the strength of randomised controlled trials-namely, randomisation to distinct interventions to isolate causality of the intervention effect compared with a control group, and minimisation of biases between the intervention and control group in terms of demographics and baseline characteristics such as body mass. We also used individual participant data, which allowed us to adjust for the same set of covariates across all studies and to perform analyses stratified by participant characteristics and not study level summaries. This also facilitated comparisons between additive and dominant FTO genotype models. Moreover, our sample size was larger, which improved the power of our meta-analysis. Our findings suggest that when robust intervention design and analysisincluding gene by treatment arm interactions, individual participant data, and a large sample size-are used, there is no evidence that FTO genotype affects weight loss in response to lifestyle or dietary interventions.

The present meta-analysis included two studies, Look AHEAD ${ }^{15}$ and DPP, ${ }^{20}$ which together contributed 


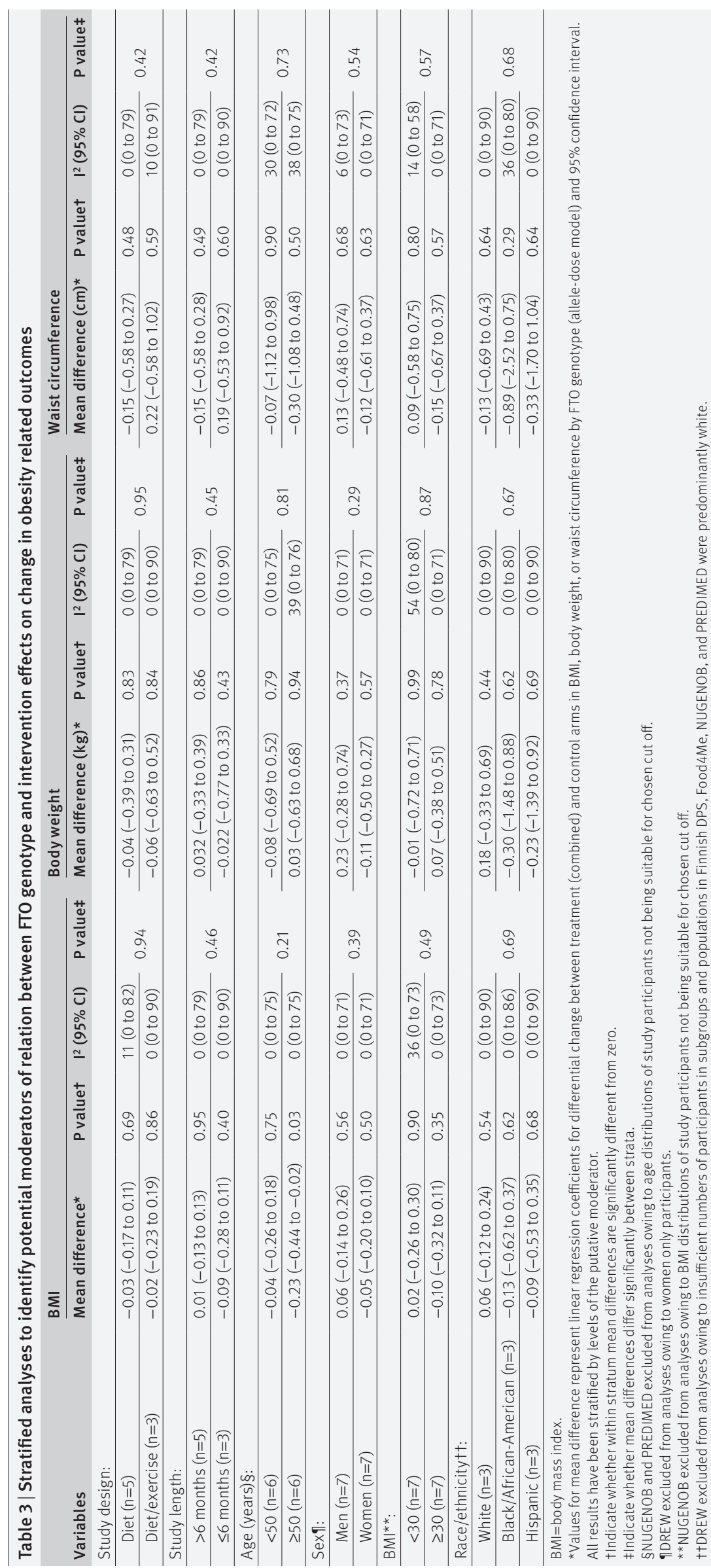

$67 \%(n=6472)$ of the total sample. Independently, neither study identified any association between FTO genotype and weight loss, ${ }^{1520}$ which was consistent with parallel and pooled analysis of both studies ${ }^{22}$ and with our overall findings. In our sensitivity analyses, omission of the largest study (Look AHEAD ${ }^{15}$ ) did not change the pattern of results, thus supporting the robustness of our findings.

Although we identified a greater reduction in body mass index for each minor allele of FTO genotype in participants aged more than 50 years, this effect was not consistent across body weight and waist circumference outcomes, suggesting that this is likely to be a chance finding. Nevertheless, recent metaregression estimates from twin studies suggest that heritability of body mass index was $0.07(\mathrm{P}=0.001)$ higher in children than in adults. ${ }^{44}$ Further research into possible subgroup effects of FTO variants and other obesity related genes is warranted to confirm or refute our findings.

Although there is good evidence that the duration of the weight loss trial influences weight lost and regained, ${ }^{45}$ with greatest loss occurring at six months followed by gradual regain, ${ }^{46}$ we did not identify any effect of study duration on the relation between FTO genotype and weight loss. Behaviour change strategies for weight management seem to have limited effect in preventing relapse. ${ }^{45}$ In a pan-European study of 742 adults, multiple single nucleotide polymorphisms were investigated for effects on weight regain over six months ad libitum diet after more than $8 \%$ weight loss, and the study investigated whether nutrient sensitive genes modified weight regain in response to glycaemic index and high protein based diets. No significant effects were found for the FTO single nucleotide polymorphisms, and although significant effects were found for several other single nucleotide polymorphisms, associations were no longer significant after adjustment for multiple testing. ${ }^{47}$ Furthermore, although a more recent study found evidence for the specific role of FTO genotype in predicting maintenance of body weight, ${ }^{40}$ results were based on a smaller ( $n=128)$, women only sample, and so the findings should be interpreted with caution. Given the lack of effective strategies for preventing weight regain and the evidence for an interaction between FTO genotype and physical activity on risk of obesity, ${ }^{48}$ further research into the role of obesity related genes and their interactions with diet and physical activity on long term weight management are needed. Although the role of FTO, and other obesity associated genes, in weight maintenance was outside the scope of the present meta-analysis, it is an important consideration for maximising the long term health benefits of weight loss and should be considered in future research.

Weight loss in response to a lifestyle intervention requires negative energy balance to be sustained over a considerable period. This process is complex and involves behaviour changes in either or both, food consumption (energy intake) and physical activity (energy expenditure). The mechanisms through which the FTO genotype could influence such processes are not known ${ }^{49}$ and might be different from those through 
which the FTO genotype influences the development of excess adiposity.

\section{Strength and limitations of this review}

A strength of this meta-analysis is that we jointly analysed individual participant data from eight randomised controlled trials using a standardised analysis strategy. Application of this standardised statistical analysis mitigated differences in study methodologies, utilised data independent of statistical significance or how results were reported initially, and improved the overall reliability of the results. Moreover, our analysis included only randomised controlled trials, considered the ideal study design by avoiding many biases and confounding effects, and this approach allowed us to observe whether any differential effect of FTO genotype on weight loss is specific to weight loss interventions by comparing weight loss between participants randomised to treatment arms and those randomised to the control arm. A further strength of our study was our ability to assess the effect of the FTO genotype on multiple measures of adiposity. Lastly, our study addresses the genetics and gene-lifestyle interactions of weight loss, a problem of public health importance, and fills a major gap in the literature on the putative role of FTO genotype in modulating weight loss in response to weight loss interventions.

An important limitation is that we evaluated the effect of FTO genotype only and, given that obesity risk and weight loss is influenced by multiple genes, ${ }^{50}$ the effect of other obesity related genes, such as MC4R and TMEM18, on weight loss in response to intervention remains to be determined. None the less, our findings are in line with those from the DPP and Finnish DPS trials, where participants with greater genetic risk for several known type 2 diabetes benefitted from lifestyle intervention equally well compared with those with lower risk. ${ }^{5152}$ Furthermore, we found a relatively small number of randomised controlled trials reporting weight loss by FTO genotype, all of which originated from North America and Europe, with predominantly white participants, which limited our power to investigate differential effects between ethnicities. Given evidence that the relation between FTO genotype and obesity varies by ethnicity, ${ }^{53}$ further well powered studies on this topic in ethnically different populations are warranted. In addition, we applied the same body mass index cut points for adiposity classification in Asian and other individuals, ${ }^{54}$ but, because Asians made up less than $5 \%$ of our total sample, this is unlikely to have impacted on our results. Given the evidence that both obesity risk and weight loss are modified by multiple genetic variants, ${ }^{55}$ our findings for FTO genotype should not be considered in isolation. A further limitation is that the present analysis did not account for the diversity of intervention designs, where the type of diet or exercise intervention may induce differential weight loss through effects on metabolism, appetite, and thermogenesis ${ }^{56}$ although we found no interaction between intervention type (diet versus exercise) and FTO genotype on weight loss. In addition, weight loss interventions may be biased by higher dropout rates than in other randomised controlled trials, ${ }^{57}$ which may have influenced our ability to identify a relation between FTO genotype and weight loss. Lastly, the authors of three studies identified in the systematic review did not agree to provide individual participant or aggregate data and thus were excluded from the present analyses. These studies were small (total participants $\mathrm{n}=441$ ) and thus their exclusion is unlikely to have affected our conclusions.

\section{Implications of the findings}

We found that the FTO genotype had no detectable effect on weight loss in overweight and obese adults in response to intervention. Importantly, our findings show that the genetic predisposition to obesity associated with the FTO minor allele can be at least partly counteracted through dietary, exercise, or drug based weight loss interventions and that those carrying the minor allele respond equally well to such interventions. Moreover, our results suggest that screening for the FTO genotype in routine clinical work would not predict weight loss success. Future public health strategies for the management of obesity should aim to induce long term improvements in lifestyle behaviours, principally eating patterns and physical activity, since these will be effective in achieving sustained weight loss irrespective of FTO genotype.

\section{Conclusions}

This systematic review and meta-analysis of individual participant data reveals that carriage of the FTO minor allele, associated with risk of obesity in the general population as well as baseline adiposity in the present study, was not associated with changes in body mass index, body weight, or waist circumference in response to weight loss intervention. Our findings show that weight loss in those carrying the FTO minor allele is similar to the rest of the population after dietary, exercise, or drug based interventions. Future studies should investigate the possibility that a panel of genetic variants, including other FTO genotypes, may modulate weight loss in obese people in response to lifestyle and other interventions.

\section{AUTHOR AFFILIATIONS}

${ }^{1}$ Human Nutrition Research Centre, Institute of Cellular Medicine, Newcastle University, Newcastle upon Tyne NE4 5PL, UK

2Deakin University, Institute for Physical Activity and Nutrition, School of Exercise and Nutrition Sciences, Victoria, Australia ${ }^{3}$ BHF Glasgow Cardiovascular Research Centre, Institute of Cardiovascular and Medical Science, University of Glasgow, Glasgow, UK

${ }^{4}$ Department of Biostatistics, Brown University School of Public Health, Providence, RI, USA

${ }^{5}$ Diabetes Unit and Center for Human Genetic Research, Massachusetts General Hospital, Boston, MA, USA

${ }^{6}$ Programs in Metabolism and Medical and Population Genetics, Broad Institute of Harvard and MIT, Cambridge, MA, USA

${ }^{7}$ George Washington University Department of Epidemiology and Biostatistics The Biostatistics Center, Rockville, MD, USA

${ }^{8}$ Department of Preventive Medicine and Public Health, University of Navarra, Pamplona, Spain 
${ }^{9}$ CIBER Fisiopatologia de la Obesidad y Nutricion and PREDIMED Network from Instituto de Salud Carlos III Spanish Government, Spain

${ }^{10}$ Department of Nutrition, Food Science and Physiology, University of Navarra, Pamplona, Spain

${ }^{11}$ Department of Epidemiology, School of Public Health and Tropical Medicine, Tulane University, New Orleans, LA, USA

${ }^{12}$ Epidemiology Domain, Saw Swee Hock School of Public Health, National University of Singapore, Singapore

${ }^{13}$ Department of Nutrition, Harvard T.H. Chan School of Public Health, Boston, MA, USA

${ }^{14}$ Novo Nordisk Foundation Centre for Basic Metabolic Research, Section on Metabolic Genetics, Faculty of Health and Medical Sciences, University of Copenhagen, Copenhagen, Denmark

${ }^{15}$ Danish Diabetes Academy, Odense, Denmark

${ }^{16}$ Department of Exercise Science, University of South Carolina, Columbia, SC, USA

17Pennington Biomedical Research Center, Louisiana State University System, Baton Rouge, LA, USA

${ }^{18}$ Institute of Public Health and Clinical Nutrition, University of Eastern Finland, Kuopio, Finland

${ }^{19}$ Department of Medical and Clinical Genetics, University of Helsinki, Finland

20Department of Chronic Disease Prevention, National Institute for Health and Welfare, Helsinki, Finland

${ }^{21}$ Dasman Diabetes Institute, Dasman, Kuwait City, Kuwait

22Diabetes Research Group, King Abdulaziz University, Jeddah, Saudi Arabia

${ }^{23}$ Human Genomics Laboratory, Pennington Biomedical Research Center, Baton Rouge, LA, USA

${ }^{24}$ Department of Human Biology, NUTRIM School of Nutrition and Translational Research in Metabolism, Maastricht University Medical Centre +, Maastricht, Netherlands

${ }^{25}$ Department of Nutrition, Exercise and Sports, Copenhagen University, Rolighedsvej 30, Frederiksberg C, Denmark

${ }^{26}$ Institute of Preventive Medicine, Bispebjerg and Frederiksberg Hospitals, The Capital Region, Denmark

27Food Science and Physiology, Centre for Nutrition Research, University of Navarra, Pamplona, Spain

${ }^{28}$ Department of Clinical Sciences, Genetic and Molecular Epidemiology Unit, Lund University, Skåne University Hospital Malmö, Malmö, Sweden

29The Miriam Hospital and the Alpert School of Medicine, Brown University, Providence, USA

${ }^{30}$ Department of Applied Sciences, Faculty of Health and Life Sciences, Northumbria University, Newcastle upon Tyne, UK We thank Shakir Chowdhury for his assistance with the literature search.

Contributors: KML, CCM, JL, and JCM designed the research. KML, CCM, GDP, and JCM wrote the paper. KML, CCM, and GDP performed the meta-analysis for all studies. All authors contributed towards the statistical analysis, critically reviewed the manuscript during the writing process, and approved the final version to be published. KML and CCM contributed equally so are joint first authors. IL and ICM are joint last authors. KML and JCM are the guarantors for the study.

Funding: KML is funded by the Alfred Deakin postdoctoral research fellowship. CCM was supported by the UK Research Councils' Lifelong Health and Wellbeing Initiative in partnership with the Department of Health (MR/K025090/1).

Competing interests: All authors have completed the ICMIE uniform disclosure form at www.icmje.org/coi_disclosure.pdf (available on request from the corresponding author) and declare: no support from companies for the submitted work; no relationships with companies that might have an interest in the submitted work in the previous three years; no spouses, partners, or children have no financial relationships that may be relevant to the submitted work; no non-financial interests that may be relevant to the submitted work.

Ethical approval: Not required.

Data sharing: No additional data available.

Transparency: The lead author (JCM) affirms that the manuscript is an honest, accurate, and transparent account of the study being reported; that no important aspects of the study have been omitted; and that any discrepancies from the study as planned (and, if relevant, registered) have been explained.
This is an Open Access article distributed in accordance with the Creative Commons Attribution Non Commercial (CC BY-NC 3.0) license, which permits others to distribute, remix, adapt, build upon this work non-commercially, and license their derivative works on different terms, provided the original work is properly cited and the use is non-commercial. See: http://creativecommons.org/licenses/ by-nc/3.0/.

1 Sepúlveda J. Murray C. The state of global health in 2014. Science 2014:345:1275-8doi:10.1126/science.1257099.

$2 \mathrm{Ng}$ M, Fleming T, Robinson M, et al. Global, regional and nationa prevalence of overweight and obesity in children and adults 1980-2013: A systematic analysis. Lancet 2014:384:76681doi:10.1016/S0140-6736(14)60460-8.

3 Celis-Morales C, Lara J, Mathers JC. Personalising nutritional guidance for more effective behaviour change. Proc Nutr Soc 2014:12:1-9.

4 Bray GA, Frühbeck G, Ryan DH, et al. Management of obesity. Lancet.

5 Willyard C. Heritability: The family roots of obesity. Nature 2014:508:S58-60doi:10.1038/508S58a.

6 Locke AE, Kahali B, Berndt SI, et al. Genetic studies of body mass index yield new insights for obesity biology. Nature 2015;518:197206doi:10.1038/nature14177.

7 Speliotes EK, Willer CI, Berndt SI, et al. Association analyses of 249,796 individuals reveal 18 new loci associated with body mass index. Nat Genet 2010;42:937-48doi:10.1038/ng.686.

8 Lindgren CM, Heid IM, Randall IC, et al. Genome-Wide Association Scan Meta-Analysis Identifies Three Loci Influencing Adiposity and Fat Distribution. PLoS Genet 2009;5:e1000508doi:10.1371/journal. pgen.1000508.

9 Yeo GSH. The role of the FTO (Fat Mass and Obesity Related) locus in regulating body size and composition. Mol Cell Endocrinol 2014;397:34-41doi:10.1016/j.mce.2014.09.012.

10 Loos RIF, Lindgren CM, Li S, et al. Common variants near MC4R are associated with fat mass, weight and risk of obesity. Nat Genet 2008;40:768-75doi:10.1038/ng.140

11 Liu C-T, Young KL, Brody I, et al. Sequence Variation in TMEM18 in Association with Body Mass Index: The Cohorts for Heart and Aging Research in Genomic Epidemiology (CHARGE) Targeted Sequencing Study. Circ Cardiovasc Genet 2014;7:344-9doi:10.1161/ CIRCGENETICS.13.000067.

12 Frayling TM, Timpson NJ, Weedon MN, et al. A Common Variant in the FTO Gene Is Associated with Body Mass Index and Predisposes to Childhood and Adult Obesity. Science 2007;316:889-94doi:10.1126 science.1141634.

13 Razquin C, Martinez JA, Martinez-Gonzalez MA, et al. A 3-year intervention with a Mediterranean diet modified the association between the rs9939609 gene variant in FTO and body weight changes. Int J Obes 2010;34:266-72doi:10.1038/ijo.2009.233.

14 Mitchell JA, Church TS, Rankinen T, et al. FTO genotype and the weigh loss benefits of moderate intensity exercise. Obesity (Silver Spring) 2010;18:641-3doi:10.1038/oby.2009.311.

15 McCaffery JM, Papandonatos GD, Huggins GS, et al. FTO predicts weight regain in the Look AHEAD clinical trial. Int) Obes 2013:37:1545-52doi:10.1038/iijo.2013.54

16 Zhang X, Qi Q, Zhang C, et al. FTO genotype and 2-year change in body composition and fat distribution in response to weight-loss diets: The POUNDS LOST trial. Diabetes 2012;61:3005-11doi:10.2337/ db11-1799.

17 Grau K, Hansen T, Holst C, et al. Macronutrient-specific effect of FTO rs9939609 in response to a 10-week randomized hypo-energetic diet among obese Europeans. Int J Obes 2009:33:1227-34doi:10.1038/ ijo.2009.159.

18 Celis-Morales C, Marsaux CFM, Livingstone KM, et al. Physical activity attenuates the effect of the FTO genotype on obesity traits in European adults: The Food4Me study. Obesity (Silver Spring) 2016;24:962-9doi:10.1002/oby.21422.

19 Livingstone KM, Celis-Morales C, Navas-Carretero S, et al. Fat mass- and obesity-associated genotype, dietary intakes and anthropometric measures in European adults: the Food4Me study. $\mathrm{Br}$ J Nutr 2016;115:440-8doi:10.1017/S0007114515004675.

20 Franks PW, Jablonski KA, Delahanty LM, et al. Assessing genetreatment interactions at the FTO and INSIG2 loci on obesity-related traits in the Diabetes Prevention Program[Erratum appears in Diabetologia. 2009 Jul;52(7):1456]. Diabetologia 2008:51:221423doi:10.1007/s00125-008-1158-x

21 Lappalainen TJ, Tolppanen AM, Kolehmainen M, et al. The common variant in the FTO gene did not modify the effect of lifestyle changes on body weight: The finnish diabetes prevention study. Obesity (Silver Spring) 2009;17:832-6doi:10.1038/oby.2008.618.

22 Papandonatos GD, Pan Q, Pajewski NM, et al. Genetic Predisposition to Weight Loss and Regain With Lifestyle Intervention: Analyses From the Diabetes Prevention Program and the Look AHEAD Randomized Controlled Trials. Diabetes 2015;64:4312-21doi:10.2337/db15-0441.

23 Xiang L, Wu H, Pan A, et al. FTO genotype and weight loss in diet and lifestyle interventions: a systematic review and meta-analysis. Am J Clin Nutr 2016;103:1162-70doi:10.3945/ajcn.115.123448. 
24 Higgins JPT, Green S. Cochrane Collaboration. Cochrane handbook for systematic reviews of interventions. Wiley-Blackwell, 2008doi:10.1002/9780470712184.

25 University of York. NHS Centre for Reviews \& Dissemination. Systematic reviews : CRD's guidance for undertaking reviews in health care.Centre for Reviews and Dissemination, University of York, 2009.

26 Moher D, Liberati A, Tetzlaff J, et al. Preferred reporting items for systematic reviews and meta-analyses: the PRISMA statement. BMJ 2009;339:b2535doi:10.1136/bmj.b2535.

27 The InterTASC Information Specialists' Sub-Group. Search filter resource 2015. www.york.ac.uk/inst/crd/intertasc/.

28 Riley RD, Lambert PC, Abo-Zaid G. Meta-analysis of individual participant data: rationale, conduct, and reporting. $B M$ 2010;340:c221. doi:10.1136/bmi.c221.

29 Borenstein M, Hedges LV, Higgins JPT, et al. A basic introduction to fixed-effect and random-effects models for meta-analysis. Res Synth Methods 2010;1:97-111doi:10.1002/jrsm.12.

30 Higgins JP, Green S. Cochrane Collaboration. Cochrane handbook for systematic reviews of interventions. Wiley-Blackwell, 2008doi:10.1002/9780470712184

31 Higgins JPT, Thompson SG, Deeks JJ, et al. Measuring inconsistency in meta-analyses, 2003.

32 loannidis JPA, Patsopoulos NA, Evangelou E. Uncertainty in heterogeneity estimates in meta-analyses. BMJ 2007;335:914 6doi:10.1136/bmj.39343.408449.80.

33 Higgins JPT, Thompson SG. Quantifying heterogeneity in a meta-analysis. Stat Med 2002;21:1539-58doi:10.1002/sim.1186.

34 Hayashino Y, Noguchi Y, Fukui T. Systematic Evaluation and Comparison of Statistical Tests for Publication Bias. J Epidemiol 2005;15:235-43doi:10.2188/jea.15.235.

35 Egger M, Smith GD, Schneider M, et al. Bias in meta-analysis detected by a simple, graphical test. BM/ 1997;315:629-34doi:10.1136/ bmj.315.7109.629.

36 Celis-Morales C, Livingstone KM, Marsaux CFM, et al. Design and baseline characteristics of the Food4Me study: a web-based randomised controlled trial of personalised nutrition in seven European countries. Genes Nutr 2015;10:450doi:10.1007/ s12263-014-0450-2.

37 Ramos RB, Casanova GK, Spritzer PM. Fat mass and obesityassociated gene polymorphisms do not affect metabolic response to hormone therapy in healthy postmenopausal women. Eur / Obstet Gynecol Reprod Biol 2012;165:302-6doi:10.1016/j. ejogrb.2012.07.024

38 de Luis DA, Aller R, Izaola $\mathrm{O}$, et al. Evaluation of weight loss and adipocytokines levels after two hypocaloric diets with different macronutrient distribution in obese subjects with rs9939609 gene variant. Diabetes Metab Res Rev 2012;28:663-8doi:10.1002/ dmrr.2323.

39 Frankwich KA, Egnatios J, Kenyon ML, et al. Differences in Weight Loss Between Persons on Standard Balanced vs Nutrigenetic Diets in a Randomized Controlled Trial. Clin Gastroenterol Hepatol 2015;13:1625-32.e1doi:10.1016/j.cgh.2015.02.044

40 Matsuo T, Nakata Y, Hotta K, et al. The FTO genotype as a useful predictor of body weight maintenance: Initial data from a 5-year follow-up study. Metabolism 2014;63:912-7doi:10.1016/j. metabol.2014.03.013.

41 Rauhio A, Uusi-Rasi K, Nikkari ST, et al. Association of the FTO and ADRB2 genes with body composition and fat distribution in obese women. Maturitas 2013;76:165-71doi:10.1016/i. maturitas.2013.07.004
42 Verhoef SPM, Camps SGJA, Bouwman FG, et al. Genetic predisposition, dietary restraint and disinhibition in relation to short and long-term weight loss. Physiol Behav 2014;128:247-51doi:10.101 6/j.physbeh.2014.02.004

43 Woehning A, Schultz JH, Roeder E, et al. The A-allele of the common FTO gene variant rs9939609 complicates weight maintenance in severe obese patients. Int J Obes 2013;37:135-9doi:10.1038/ ijo.2012.14

44 Elks CE, den Hoed M, Zhao JH, et al. Variability in the Heritability of Body Mass Index: A Systematic Review and Meta-Regression. Front Endocrinol (Lausanne) 2012;3:29doi:10.3389/fendo.2012.00029.

45 Turk MW, Yang K, Hravnak M, et al. Randomized Clinical Trials of Weight-Loss Maintenance: A Review. J Cardiovasc Nurs 2009;24:5880doi:10.1097/01.JCN.0000317471.58048.32.

46 Jeffery RW, Drewnowski A, Epstein LH, et al. Long-term maintenance of weight loss: current status. Health Psychol 2000;19:5-

16doi:10.1037/0278-6133.19.Suppl1.5.

47 Larsen LH, Angquist L, Vimaleswaran KS, et al. Analyses of single nucleotide polymorphisms in selected nutrientsensitive genes in weight-regain prevention: The DIOGENES study. Am / Clin Nutr 2012;95:1254-60doi:10.3945/ajcn.111.016543.

48 Kilpeläinen TO, Qi L, Brage S, et al. Physical Activity Attenuates the Influence of FTO Variants on Obesity Risk: A Meta-Analysis of 218,166 Adults and 19,268 Children. PLoS Med 2011;8:e1001116doi:10.1371/ journal.pmed.1001116.

49 Livingstone KM, Celis-Morales C, Lara J, et al. Associations between FTO genotype and total energy and macronutrient intake in adults: a systematic review and meta-analysis. Obes Rev 2015;16:66678doi:10.1111/obr.12290

50 Goni L, Cuervo M, Milagro Fl, et al. Gene-Gene Interplay and Gene-Diet Interactions Involving the MTNR1B rs10830963 Variant with Body Weight Loss. J Nutrigenet Nutrigenomics 2014;7:232-42doi:10.1159/000380951.

51 Uusitupa MI, Stančáková A, Peltonen M, et al. Impact of Positive Family History and Genetic Risk Variants on the Incidence of Diabetes: The Finnish Diabetes Prevention Study. Diabetes Care 2011;34:41823doi:10.2337/dc10-1013.

52 Florez JC, Jablonski KA, Bayley N, et al. TCF7L2 Polymorphisms and Progression to Diabetes in the Diabetes Prevention Program. N Engl J Med 2006;355:241-50doi:10.1056/NEJMoa062418.

53 Peng S, Zhu Y, Xu F, et al. FTO gene polymorphisms and obesity risk: a meta-analysis. BMC Med 2011;9:71 doi:10.1186/1741-7015-9-71

54 WHO expert consultation. Appropriate body-mass index for Asian populations and its implications for policy and intervention strategies. Lancet 2004;363:157-63doi:10.1016/S0140-6736(03)15268-3.

55 Sørensen TI, Boutin P, Taylor MA, et al. Genetic Polymorphisms and Weight Loss in Obesity: A Randomised Trial of Hypo-Energetic High- versus Low-Fat Diets. PLoS Clin Trials 2006;1:e12doi:10.1371/ journal.pctr.0010012

56 Martinez JA, Navas-Carretero S, Saris WHM, et al. Personalized weight loss strategies[mdash]the role of macronutrient distribution. Nat Rev Endocrinol 2014:10:749-60doi:10.1038/nrendo.2014.175.

57 Fabricatore AN, Wadden TA, Moore RH, et al. Attrition from Randomized Controlled Trials of Pharmacological Weight Loss Agents: A Systematic Review and Analysis. Obes Rev 2009;10:333-41doi:10.1111/j.1467-789X.2009.00567.x.

\section{Supplementary file: supplementary appendices}

